\title{
Educação nutricional nos parâmetros curriculares nacionais para o ensino fundamental
}

\author{
Nutrition education in the national curricular \\ parameters for elementary schooling
}

Maria Letícia Galluzzi BIZZO'

Lídia LEDER²

RE S U M O

A promoção de saúde entre crianças maiores de cinco anos de idade habitualmente não é prioridade nas políticas de saúde oficiais, em particular no ambiente escolar, não obstante requeiram intensivas ações nesse sentido, incluindo programas educativos em nutrição. O presente trabalho constitui uma reflexão acerca da inserção da educação nutricional como Tema Transversal nos Parâmetros Curriculares Nacionais para o Ensino Fundamental. Além de recomendar premissas pedagógicas, enfoca alguns dos principais requisitos técnico-científicos para tal implementação: a formação diferenciada do nutricionista, a compreensão da construção e mudança dos hábitos alimentares de escolares, a modelagem no contexto alimentar da escola e a integração com outras ações e intervenções. O trabalho que deu origem a este artigo motivou dois projetos de lei, ambos estabelecendo princípios e diretrizes para a educação nutricional e a segurança alimentar e nutricional de escolares, um deles tramitando na Câmara Municipal do Rio de Janeiro e o outro no Congresso Nacional.

Termos de indexação: educação em saúde, educação nutricional, escolares, nutrição, promoção de saúde.

A B S T R A C T

Health promotion among children over 5 years old has not been a priority in the Brazilian health legislation, in particular in the school environment, although schoolchildren constitute a group that requires intensive actions in this direction, including educative programs in nutrition. The present work considers aspects concerning the insertion of nutrition education as a Transversal Subject in the National Curricular Parameters for Elementary Schooling. Besides recommending pedagogical premises, it focuses on some of the main technical-scientific requirements for such an implementation: the adequacy of undergraduate courses in nutrition, the modelling and understanding of the construction and change in food habits in the school

\footnotetext{
1 Departamento de Nutrição Social e Aplicada, Instituto de Nutrição, Centro de Ciências da Saúde, Universidade Federal do Rio de Janeiro. Av. Brigadeiro Trompowsky, s/n, bloco J, sala 10, 21941-590, Rio de Janeiro, RJ, Brasil. Correspondência para/Correspondece to: M.L.G. BIZZO.E-mail: <galluzzi@acd.ufrj.br>.

2 Nutricionista, Curso de Especialização em Intervenções Nutricionais em Saúde Coletiva, Departamento de Nutrição Social e Aplicada, Instituto de Nutrição, Universidade Federal do Rio de Janeiro. Rio de Janeiro, RJ, Brasil.
} 
context, and integration with other actions and interventions. The study of which this article is an integral part has given rise to two prospective laws, establishing principles and guidelines for the nutrition education and nutritional and food safety of schoolchildren, one of these laws being processed in the City Council of Rio de Janeiro and the other in the National Congress.

Indexing terms: health education, nutrition education, school children, nutrition, health promotion.

\section{N T R O D U Ç Ã O}

A promoção de saúde no ambiente escolar vem sendo fortemente recomendada por órgãos internacionais', pois as crianças maiores de cinco anos habitualmente se acham excluídas das prioridades estratégicas das políticas oficiais de saúde, apesar de biológica, nutricional e socialmente suscetíveis². Tal promoção vem sendo apontada como medida estratégica também em virtude da recente expansão da cobertura escolar para essa faixa de idade no país, propiciando, portanto, acesso a essa população no próprio ambiente escolar ${ }^{3}$. Uma análise geral de programas de saúde escolar brasileiros demonstra que esses evocam integralidade, mas exibem prática assistencialista e subdividida em ações isoladas, reproduzindo paradigmas da saúde, o que, coadunado ao quadro epidemiológico típico ${ }^{1}$, converge para reforçar a necessidade de implementação de uma política nacional de educação nutricional do escolar.

Este trabalho tem como objetivo lançar uma reflexão acerca da inserção da educação nutricional como tema transversal nos Parâmetros Curriculares Nacionais para o Ensino Fundamental, em especial para as escolas públicas, onde se concentram parcelas populacionais mais desprivilegiadas econômica, social e culturalmente, e mais atingidas pela marginalização no atendimento de saúde ${ }^{3}$, e visa, ainda, apresentar alguns dos requisitos a tal inserção. $\mathrm{O}$ trabalho do qual este artigo deriva motivou o projeto de lei no 1587/2003, presentemente tramitando na Câmara Municipal do Rio de Janeiro, e o projeto de lei no 3310/2004, em tramitação no Congresso Nacional, ambos estabelecendo princípios e diretrizes para a educação nutricional e segurança alimentar e nutricional de escolares.

\section{Premissas pedagógicas}

Propondo-se flexíveis, inovadores, de caráter integrado e promotores de cidadania, os Parâmetros Curriculares Nacionais constituem o plano curricular oficial para o ensino fundamental brasileiro ${ }^{4}$. Além das disciplinas tradicionais, abrangem seis temas transversais: ética, pluralidade cultural, meio ambiente, saúde, orientação sexual e trabalho e consumo. Entende-se que o ensino sobre nutrição seja fundamental na promoção de saúde, que deve ter lugar na escola, e, por isso, a educação nutricional não pode deixar de compor, criticamente, um plano nacional oficial de ensino.

A política curricular expressa o grau de legitimidade e poder de decidir sobre conteúdos de aprendizagem, segundo o papel conquistado por cada ator na construção de um projeto pedagógico 5 . Ensino é decisão e ideologia a cada ato. Se em países desenvolvidos cientistas e técnicos especialistas contribuem para a formulação dos currículos escolares ${ }^{6}$, no Brasil falta a participação de vários segmentos da sociedade.

É de considerar que uma possível inserção da educação nutricional no ensino fundamental tenha mérito para se fundamentar em metodologia pedagógica que se configure: 1) dialogal, pois, no dizer de Habermas" "só podem reclamar validade as normas que encontrem o assentimento de todos os envolvidos, estando estes na condição de participantes de um discurso prático"(p.66), subentendendo-se que a linguagem dialogal é um domínio consensual de comportamentos que mutuamente se orientam em uma coordenação de ações, e não apenas em transmissão de informações ${ }^{8}$; 2) significativa, ou seja, que tenha sentido para o aluno"; 3) problematizadora, levando à reflexão sobre 
causas, mecanismos e soluções das questões nutricionais, superando uma visão ingênua por uma visão crítica e criativa, capaz de transformar o contexto vivido ${ }^{10}$; 4) transversal, fluindo em caráter integrado com as demais matérias do currículo ${ }^{11}$; 5) lúdica, sempre que adequado, como motivadora e mediadora da compreensão do real pela criança12; 6) propositivamente valendo-se de métodos construtivistas, proporcionando ao sujeito a construção própria de conhecimento, em contexto ativo e real'12; e 7) que cultive a construção de cidadania, pelo fomento de senso crítico e empreendedor.

A implementação dessa proposta não se daria livre de tensões e contradições por desafiar o modelo vigente, sendo essencial compreender, antes, o que as crianças consideram como práticas saudáveis e identificar fatores associados a seu engajamento nelas. A educação nutricional proporia a construção coletiva do conhecimento mediante planejamento didático participativo com integração entre a equipe de saúde, a escola, a criança e a família, e tendo como ponto-chave os conteúdos trabalhados ao longo e no momento da expressão das práticas, crenças, saberes e vivências da criança, de maneira integrada, e não dissociados em práticas pedagógicas exclusivamente teóricas. O conhecimento técnico-científico do profissional de saúde seria compartilhado ao ritmo da própria problematização dos conhecimentos e vivências da criança.

Quanto aos recursos didáticos de apoio, como material audiovisual, não são meros coadjuvantes e devem guardar estrita coerência com as premissas pedagógicas anteriormente referidas, constituindo-se provocativos e não meramente contemplativos, e com expressiva ênfase em imagens e esquemas didáticos que levem a pensar e não a imprimir conhecimento pronto. O treinamento dos professores é uma condição, sensibilizando-os para o engajamento, processo mediado pelo profissional de saúde como agente instrucional e motivacional. Sendo proposta descentralizadora, pede adaptação local de conteúdos e objetivos. Descentralização do ensino implica redistribuição de poder, amplificação da eficiência de gestão e ênfase na diversidade cultural, buscando romper com políticas educacionais - que nesse aspecto não muito se distanciam das de saúde - em que o Estado mantenha atada a centralização do sistema e descomprometa-se com a descentralização, subtraindo-lhe legitimação ${ }^{13}$.

A eficácia da educação nutricional ao escolar poderia estar concebida não circunscrita como simples verificação de conhecimentos, e sim evoluindo pela incorporação da avaliação de práticas e indicadores efetivos de saúde no decurso do processo educativo (avaliação de processo) e convergindo para replanejamentos de aperfeiçoamento (produto de avaliação de resultado), sinergizada por complementaridade entre variáveis quantitativas e qualitativas. O grau de informação, por si só, potencializa maior autocuidado de saúde ${ }^{1}$ e, nesse contexto, têm-se focado a "alfabetização em saúde" e a "alfabetização em nutrição", que avaliam o grau de domínio e compreensão de leigos sobre conceitos e inter-relações mínimas de saúde e nutrição, domínio esse considerado forma de empoderamento ${ }^{14}$, por instrumentalizar para conseguir saúde. Não obstante, uma avaliação restrita à mensuração de conhecimento geralmente implica exclusão. Uma avaliação global, preocupada com a aprendizagem, pressupõe acolhimento, tendo em vista a transformação.

\section{REQUISITOS TÉ C N I C O-CIEN TII F I C OS ASSOCIADOS À I M P LE M E N T A ÇÃ O DA E DUCAÇÃ O NUTRIÇIONAL}

\section{Educação nutricional do ensino fundamental}

A preparação básica do nutricionista com vistas à prática pedagógica em nutrição tem lugar majoritariamente, por meio da disciplina de graduação Educação Nutricional, que deve capacitar o profissional a atuar em todos os níveis 
desse processo educativo. Apesar disso, evidencia-se que a disciplina Educação Nutricional necessita ser fortalecida, não apenas como veículo de qualificação profissional para a compreensão e domínio de instrumentos metodológicos, mas, sobretudo, para a construção de uma capacidade criadora e analítica fundamentada em sólida formação teórica e em experiências práticas significativas. $\mathrm{O}$ atendimento a esses quesitos e a ênfase nessa formação curricular, aspectos ainda pouco sedimentados na capacitação básica do profissional, configuram-se meios de instrumentalizar nutricionistas para desempenhar papéis de grande monta na educação nutricional escolar.

Emerge ainda uma relevante faceta adicional atinente aos cursos de nutrição, no que tange às políticas de promoção de saúde escolar. As Diretrizes Curriculares Nacionais para os cursos de graduação em Nutrição, estabelecidas pelo Ministério da Educação no ano de 2001'5, vieram outorgar, em seu artigo terceiro, inciso segundo, uma nova possibilidade de formação (além daquela tradicional, de cunho generalista), que consiste na graduação sob a modalidade de licenciatura em Nutrição. Tal formação qualifica o nutricionista a atuar na educação básica e na educação profissional em Nutrição. As referidas diretrizes trouxeram ainda, em seu bojo, uma expansão das dimensões recomendadas na formação do nutricionista, fazendo imprimir a marca da aprendizagem não restrita a conteúdos, mas igualmente abrangendo habilidades $e$ competências, como as de tomada de decisões e liderança, assim como uma formação que viabilize atuar em políticas e programas de educação, visando à promoção da saúde em âmbito local, regional e nacional, bem como à atuação na formulação e execução de programas de educação nutricional. Embora seu artigo de número treze estabeleça que a graduação, sob a modalidade de licenciatura, deva ser regulamentada por resoluções complementares, exigência ainda pendente, resta patente que a possibilidade dessa formação se configura um avanço de vulto, merecendo ser fomentada como meta a ser galgada pelo nutricionista, encetando a conquista de posições em que esse profissional crie, lidere, execute e avalie políticas e ações estratégicas de educação nutricional na promoção de saúde de populações escolares.

\section{Hábitos alimentares de escolares}

Evidências indicam que os indivíduos são mais propensos a aceitar a responsabilidade sobre eventos positivos do que a se culpar por comportamentos negativos ${ }^{16}$. A Teoria da Cognição e Comportamento em Saúde ${ }^{17}$ destaca a relevância de descobrir a rede de crenças causais de doenças, inclusive para crianças; as atribuições causais são mediadoras de comportamentos em saúde e teriam três possíveis dimensões: locus, estabilidade e controlabilidade. A primeira delimita o quanto a causa é considerada, pelo indivíduo, interna (individual) ou externa (ambiental); a segunda, o grau em que é percebida por ele como estando sob seu controle, o que inclui auto-estima; a terceira, o quanto ele a considera passível de mudança. Tal teoria tem embasado estudos comportamentais em saúde infantil; na criança, essa compreensão avança com a idade e com o desenvolvimento cognitivo ${ }^{18}$.

Embora profusos em outros países e podendo contribuir substancialmente para a compreensão de causas e cenários da alimentação infantil e subsidiar políticas escolares de educação nutricional e alimentação, no Brasil há expressiva carência de estudos comportamentais sobre hábitos alimentares de pessoas dessa faixa etária. Sobeja recomendação de que variáveis biológicas, sociais e culturais sejam consideradas na formulação desses estudos tem sido uma constante; variáveis psicocognitivas mereceriam deixar de se constituir lacuna em tais investigações. Teorias comportamentais têm galgado exponencial importância nesse campo, com rigor e sofisticação ${ }^{19}$; objetivam avaliar hábitos e mudanças alimentares segundo cabedal metodológico válido, confiável, prático e sensível aos estágios de alteração ${ }^{20}$ e podem ter potencial uso na pesquisa longitudinal de hábitos alimen- 
tares, eficácia da educação nutricional, papel do alimento nas famílias, análise de tendências seculares em alimentação e nutrição, identificação de mecanismos de mediação da mudança de hábitos, correlação entre hábitos alimentares distintos, associação entre alimentação e comportamentos de autocuidado ou de não autocuidado, multiplicação ou não da educação nutricional no ambiente familiar, métodos de maior impacto na educação nutricional, iniciativas intersetoriais para acelerar o consumo de alimentos saudáveis, dentre outros.

Novas intervenções e pesquisas em hábitos alimentares têm empregado tais modelos, com fundamentação axial no indivíduo ou em comunidades, modulados para saúde pública ${ }^{19} \mathrm{e}$ focando o desenvolvimento, manutenção e mudança de padrões de consumo alimentar e de formas de aquisição, preparo e consumo dos alimentos, mais do que extraindo retratos de uma estrutura de padrão dietético de curto prazo e descontextualizada social e psicologicamente; tanto a mensuração quanto a alteração de comportamentos dietéticos circunscrevem-se como dinâmicas e complexas, e a mudança de hábitos alimentares requer, tanto mais do que prescrição ou proibição de alimentos, sua compreensão20.

\section{Modelagem na aceitação do alimento}

No contexto escolar parece relevante exibir e estimular o consumo de alimentos saudáveis e não comercializar alimentos prejudiciais; há evidências de que a disponibilidade de alimentos em lanchonetes influencia hábitos alimentares e não o contrário ${ }^{21}$. Manipular, preparar e provar alimentos pode estimular seu consumo ${ }^{22}$. Gostar de frutas e legumes é, logicamente, um fator mais relevante de determinação do consumo de tais alimentos entre escolares do que a ingestão por parte de seus pais ou suas atitudes em relação à alimentação dos filhos ${ }^{22}$, porém, no ambiente escolar há ainda muitas variáveis a serem estudadas.
Pesquisas em outros países analisam o papel do professor como modelo de estímulo à aceitação de alimentos no ambiente escolar; alguns desses estudos chegaram à conclusão de que, de fato, é relevante o papel do professor nessa modelagem ${ }^{23}$, porém outras investigações deduziram ser essa modelagem relativa ou pequena ${ }^{24}$, sendo mais importante do que ela o papel exercido por colegas de similar faixa etária ${ }^{25}$.

Distintas situações de encorajamento do consumo de alimentos saudáveis na escola têm sido avaliadas: professor consumindo os alimentos da merenda escolar; professor consumindo e expressando satisfação, estímulo para que a criança prove um alimento; oferecimento de opções de escolha dentro do mesmo grupo de alimentos; simples exposição de alimentos saudáveis; uso exploratório do olfato; sobremesa como recompensa; diversas condições ambientais no refeitório; oferecimento de alimentos novos e comparação com a aceitabilidade de alimentos anteriormente oferecidos; aceitação imediata e de longo prazo; estímulo através da verbalização de atributos positivos do alimento; e simples ingestão de alimentos saudáveis, por professores ou funcionários, na presença do escolar, sem manifestações verbais ${ }^{23}$.

Alguns estudos apontam que, para alimentos novos, a criança gradualmente alcançaria confiança em aceitar influência ${ }^{25}$, e outros estudam a neofobia alimentar, uma aversão, freqüente em crianças, a experimentar novos alimentos ${ }^{23}$. Em alguns países, acham-se validados instrumentos diagnósticos como a Escala de Atitude Alimentar ${ }^{26}$ e a Escala de Avaliação de Neofobia Alimentar ${ }^{27}$, e há ampla gama de leis e programas oficiais que contam com expressiva cobertura, enfática sensibilização e treinamento, bem como acurado detalhamento de educação nutricional escolar, assim como ações concretas de amplitude populacional para o desenvolvimento de hábitos, no longo prazo, de compra, preparo e manutenção de consumo saudáveis de alimentos ${ }^{28}$. 


\section{Integração com outras ações e intervenções}

Ações integradas de saúde sinergizariam o impacto da educação nutricional ao escolar; para citar algumas: políticas nacionais adequadas de alimentação e nutrição, talvez incluindo legislação regulatória para alimentos prejudiciais; interlocução do programa de merenda escolar com a atenção primária; efetiva monitoração por Sistema de Vigilância Alimentar e Nutricional; suporte à referenciação para atendimento nutricional individualizado; modelos locais personalizados de educação nutricional; integração com o Programa de Saúde da Família e instituições Amigas da Criança; treinamento dos manipuladores da merenda, possibilitando, no mínimo, redução de sódio e lipídios das refeições; incentivo e integração com iniciativas comunitárias que guardem conexão com programas escolares de saúde; e contínua ênfase em pesquisa metodológica de avaliação, desenvolvimento e validação de meios consistentes e viáveis de educação nutricional escolar com desenhos experimentais e interventivos sólidos, inovadores e participativos.

\section{CONCLUSÃO}

A implementação da educação nutricional como obrigatória no ensino fundamental evidencia-se como uma política pública premente, essencial às necessidades nutricionais, de saúde e sociais da população escolar, demandando investimentos em sua concretização e nos requisitos técnico-científicos fundamentais à sua efetivação.

\section{REFERÊ NCIAS}

1. Organización Pan-Americana de la Salud. Rede Latinoamericana de Escuelas Promotoras de la Salud. Washington (DC): FAO; 1999.

2. Fundo das Nações Unidas para a Infância. Situação mundial da infância 1998. Brasília: Unesco; 1998.
3. Cyrino EG, Pereira MLT. Reflexões sobre uma proposta de integração saúde-escola: o projeto saúde e educação de Botucatu, São Paulo. Cad Saúde Pública. 1999; 15(Supl 2):39-44.

4. Brasil. Ministério da Educação e do Desporto. Secretaria do Ensino Fundamental. Parâmetros curriculares nacionais 1996 [Internet] [acesso 2002 set 20]. Disponível em: http://www.mec.gov.br/sef/ pen.shtm

5. Pacheco JP. Políticas curriculares descentralizadas: autonomia ou recentralização? Educ Soc. 2000; 21(73):139-61.

6. Bizzo MLG. Difusão científica, comunicação e saúde. Cad Saúde Pública. 2002; 18(1):307-14.

7. Habermas J. Moral consciousness and communicative action. Cambridge: Polity Press; 1990. p.66

8. Maturana HR, Varela FJ. Autopoiesis and cognition: the realization of the living. Reidel: Dordrecht; 1980.

9. Ausubel D, Novak J, Hanesian H. Educational psychology: a cognitive view. 2nd ed. New York: Holt, Rinehart \& Winston; 1978. p.33-46.

10. Freire P. Conscientização: teoria e prática da libertação. 3.ed. São Paulo: Moraes; 1980.

11. Gallo S. Transversalidade e educação: pensando uma educação não-disciplinar. In: Alves NE, Garcia $\mathrm{RL}$, organizadores. O sentido da escola. Rio de Janeiro: DPA; 1999. p.17-41.

12. Piaget J. A linguagem e o pensamento da criança. Rio de Janeiro: Fundo de Cultura; 1959.

13. Weiler $H$. Enfoques comparados en descentralización educativa. In: Pereyra M, organizador. Globalización y descentralización de los sistemas educativos. Barcelona: Pomares Ediciones; 1996. p.74-90.

14. Fourez G. Scientific and technological literacy as a social practice. Social Studies Sci. 1997; 27(6):903-22

15. Brasil. Ministério da Educação. Conselho Nacional de Educação [Internet] [acesso 2004 jun 18]. Câmara de Educação Superior. Resolução CNE/CES 05/2001: Diretrizes curriculares nacionais para o curso de graduação em Nutrição. Disponível em: http://www.mec.gov. br/cne/pdf/CES05.pdf

16. Whitley Jr BE, Frieze IH. Children's causal attribution for success and failure in achievement settings: a meta analysis. J Educ Psychol. 1985; 77(5):608-16.

17. Weiner B. An attributional theory of achievement motivation and emotion. Psychol Rev. 1985; 92(4):548-73. 
18. Walsh ME, Bibace R. Children's conceptions of AIDS: a developmental analysis. J Pediatr Psychol. 1991; 16(3):273-85.

19. Glanz K. Behavioral research contributions and needs in cancer prevention and control: dietary change. Prev Med. 1997; 26 Suppl 1:S43-55.

20. Kristal AR, Beresford SA, Lazovich D. Assessing change in diet intervention research. Am J Clin Nutr. 1994; 59 Suppl 1:S185-9.

21. Edmonds J, Baranowski T, Baranowski J, Cullen KW, Myres D. Ecological and socioeconomic correlates of fruit, juice, and vegetable consumption among African-American boys. Prev Med. 2001; 32(6):476-81.

22. Guthrie CA, Rapoport L, Wardle J. Young children's food preferences: a comparison of three modalities of food stimuli. Appetite. 2000; 35(1):73-7.

23. Bandura A. Self-efficacy: the exercise of control. New York: Freeman \& Co; 1997.

24. Hendy HM, Raudenbush B. Effectiveness of teacher modeling to encourage food acceptance in preschool children. Appetite. 2000; 34(1):61-76.

25. Harris JR. Where is the child's environment: a group socialization theory of development. Psychol Rev. 1995; 102(3):458-89.

26. Raudenbush $B$, van der Klaauw NJ, Frank RA. The contribution of psychological and sensory factors to food preference patterns as measured by the Food Attitudes Survey (FAS). Appetite. 1995; 25(1):1-15.

27. Loewen R, Pliner P. The Food Situations Questionnaire: a measure of children's willingness to try novel foods in stimulating and nonstimulating situations. Appetite. 2000; 35(3):239-50.

28. Gorder DD, Dolecek TA, Coleman GG. Dietary intake in the multiple risk factor intervention trial (MRFIT): nutrient and food group changes over six years. J Am Diet Assoc. 1986; 86(6):744-51.

Recebido para publicação em 18 de julho de 2003 e aceito em 17 de outubro de 2004 